\title{
Excitonic and Polaronic Properties of 2D Hybrid Organic-Inorganic Perovskites
}

Jun Yin,1,2 Hong Li, , Daniele Cortecchia, , Cesare Soci, 2 and Jean-Luc Brédas 1,*

1 Laboratory for Computational and Theoretical Chemistry of Advanced Materials, Division of Physical Science and Engineering, King Abdullah University of Science and Technology, Thuwal 23955-6900, Kingdom of Saudi Arabia

2 Division of Physics and Applied Physics, School of Physical and Mathematical Sciences, Nanyang Technological University, 21 Nanyang Link, Singapore 637371

3 Energy Research Institute@NTU (ERI@N), Research Techno Plaza, Nanyang Technological University, 50 Nanyang Drive, Singapore 637553

\section{Corresponding Author}

jean-luc.bredas@kaust.edu.sa; jean-luc.bredas@chemistry.gatech.edu 


\begin{abstract}
We theoretically characterize the unusual white-light emission properties of two-dimensional (2D) hybrid organic-inorganic perovskites with an $\mathrm{APbX}_{4}$ structure (where $\mathrm{A}$ is a bidentate organic cation and $\mathrm{X}=\mathrm{Cl}, \mathrm{Br}$ ). In addition to band-structure calculations including corrections due to spin-orbit couplings and electron-hole interactions, a computationally intensive molecular-cluster approach is exploited to describe the excitonic and polaronic properties of these $2 \mathrm{D}$ perovskites at the atomistic level. Upon adding or removing an electron from the neutral systems, we find that strongly localized small polarons form in the $2 \mathrm{D}$ clusters. The polaron charge density is distributed over just $\sim 1.5$ lattice sites, which is consistent with the calculated large polaron binding energies, on the order of $\sim 0.4-1.2 \mathrm{eV}$.
\end{abstract}

\title{
TOC GRAPHICS
}

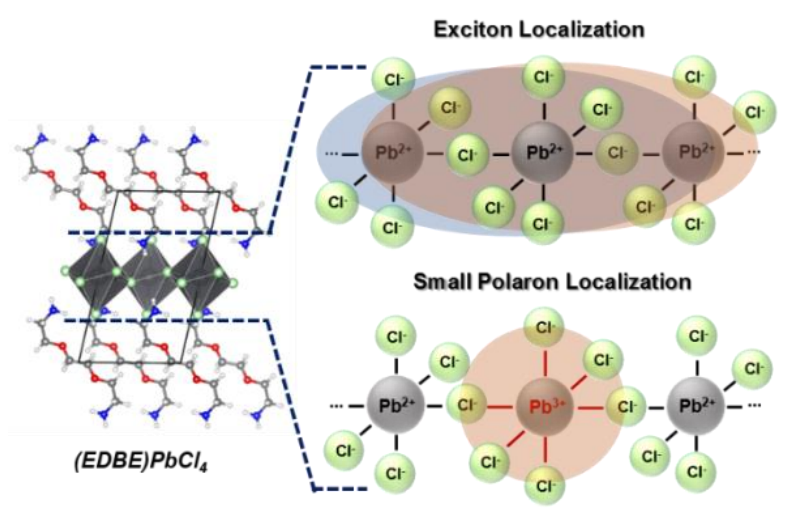


Over the past few years, three-dimensional (3D) hybrid organic-inorganic perovskites with general formula $\mathrm{APbX}_{3}$ (where $\mathrm{A}$ is a monovalent cation and $\mathrm{X}=\mathrm{Cl}, \mathrm{Br}$, I) have demonstrated outstanding optoelectronic properties that have led to thin-film solar cell and light-emitting diode applications.1-3 Hybrid perovskites with low-dimensionality also display remarkable electronic and optical characteristics.4 The reduction in dimensionality, such as in the case of layered twodimensional (2D) perovskites $\mathrm{APbX}_{4}$, leads to strong quantum/dielectric confinement effects responsible for significant changes in the optical properties.5-9 A particularly interesting feature is the broadband, white-light photoluminescence (PL) at room temperature shown by a series of $2 \mathrm{D}$ perovskites such as $(\mathrm{API}) \mathrm{PbBr}_{4} \quad(\mathrm{API}=N-(3$-aminopropyl)imidazole), 10 (EDBE) $\mathrm{PbX} 4$ (EDBE=2,2-(ethylenedioxy)bis(ethylammonium), $\mathrm{X}=\mathrm{Cl}, \mathrm{Br}), 11\left(\mathrm{C}_{6} \mathrm{H}_{11} \mathrm{NH}_{3}\right)_{2} \mathrm{PbBr}_{4}, 12$ and $(\mathrm{N}-$ MEDA $)\left[\mathrm{PbBr}_{4-\mathrm{x}} \mathrm{Cl}_{\mathrm{x}}\right] \quad(\mathrm{N}-\mathrm{MEDA}=\mathrm{N} 1-$ methylethane-1,2-diammonium $) 13$. While earlier works attributed the origin of such a broadband photoluminescence either to deep-trap surface states 14-15 or to energy transfer from the inorganic to the organic layers, 10 the formation of self-trapped excitons is now generally accepted as the main mechanism underlying the large Stokes shift and emission broadening.11-13, 16-17 Similarly, it has been proposed that self-trapping phenomena could affect the homogeneous PL broadening of 3D MAPbI3,18 where the existence of small polarons triggered by the collective reorientation of MA+ cations was theoretically predicted.19

Recently, a first-principles study of exciton self-trapping in 2D hybrid perovskites has shown that local perturbations of specific crystal lattice sites induce the localization of self-trapped species in the perovskite inorganic framework, and result in the formation of small polarons.17 However, the standard computational methods used in that work, relying on the local perturbation of lattice parameters within a supercell, do not allow the assessment of the actual 
charge redistributions induced by the geometric lattice relaxations,20-23 in particular those related to self-trapped excitons or small polarons. Here, we adopt a computationally intensive approach based on molecular clusters in order to provide a quantitative description of the excitonic and polaronic characteristics of the 2D white-light emitting hybrid perovskites (EDBE) $\mathrm{PbCl}_{4}$ and (EDBE) $\mathrm{PbBr} 4$. We first show that the electronic bands and optical transitions of these 2D perovskites are well reproduced via explicit consideration of the electron-hole interactions, which result in exciton wavefunctions that are highly localized within the inorganic framework. Then, we provide a direct comparison between the small-polaron species predicted via local perturbations of the perovskite crystal lattice, and those obtained from the rigorous charge density distributions evaluated in the molecular clusters. The molecular-cluster approach underlines the charge-vibration coupling effects that lead to the formation of molecular polarons. Our results afford a robust description of exciton self-trapping in 2D perovskites, and point to the applicability of our methodology to the study of perovskites with different compositions and varying dimensionality.

From a structural point of view, the inorganic framework of the 2D layered perovskites can be derived by slicing the global perovskite structure along specific crystallographic planes. As shown in Figures 1a-b, in the case of (EDBE) $\mathrm{PbCl}_{4}$, the flat inorganic layers are formed by cuts along the (100) direction, while for (EDBE) $\mathrm{PbBr}_{4}$, the rippled inorganic layers are generated along the (110) direction. In contrast to the small organic cations in 3D perovskites (e.g., $\mathrm{CH}_{3} \mathrm{NH}_{3+}$ in $\mathrm{MAPbI}_{3}$ ), which can freely rotate, the EDBE cations are relatively rigid and thus help in locking the crystal structure in these preferred orientations. Figures $1 \mathrm{c}-\mathrm{d}$ show the experimental absorption and photoluminescence spectra of (EDBE) $\mathrm{PbX}_{4}(\mathrm{X}=\mathrm{Cl}$ or $\mathrm{Br})$ thin films 
at room temperature. These samples were synthesized by spin-coating the precursors (EDBE) $\mathrm{X}_{2}$ and $\mathrm{PbX}_{2}$ (the detailed synthetic methods can be found in Refs 11, 17). (EDBE) $\mathrm{PbCl}_{4}$ and (EDBE) $\mathrm{PbBr} 4$ both show a sharp excitonic absorption peak, at $3.72 \mathrm{eV}$ and $3.32 \mathrm{eV}$, respectively, which is related to strong quantum confinement effects. Moreover, the better isolation of the inorganic quantum-well layers due to the significant length of the EDBE cations, together with the much lower dielectric constant within the organic cation layers, lead to substantial exciton binding energies: $360 \mathrm{meV}$ for (EDBE) $\mathrm{PbCl}_{4}$ and $330 \mathrm{meV}$ for (EDBE) $\mathrm{PbBr}_{4}$, as deduced recently from low-temperature absorption spectra.17 These values are much larger than in $3 \mathrm{D}$

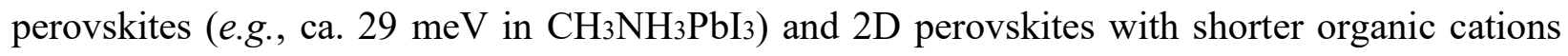
(e.g., in the range of $60-181 \mathrm{meV}$ in $\left.\left(\mathrm{CH}_{3} \mathrm{NH}_{3}\right)_{n}\left(\mathrm{C}_{9}\right)_{n} \mathrm{~Pb}_{n} \mathrm{I}_{2 \mathrm{n}+1}(\mathrm{n}=2-4)\right)$. The absorption above $4.13 \mathrm{eV}$ can be attributed to the onset of band-edge and higher-energy inter-band transitions.24 Using an ultraviolet excitation wavelength tuned at the exciton peaks, both (EDBE) $\mathrm{PbCl}_{4}$ and (EDBE) $\mathrm{PbBr}_{4}$ show broadband white-light emission spanning the entire visible range, and large Stokes shifts $(\sim 1 \mathrm{eV})$; this is in contrast with the narrow emission spectra and small Stokes shifts typical of excitonic states in 2D hybrid perovskites like (PEA) ${ }_{2} \mathrm{PbX} 425$ or $(\mathrm{ODA})_{2} \mathrm{PbX}_{4.26}$ 
(a)

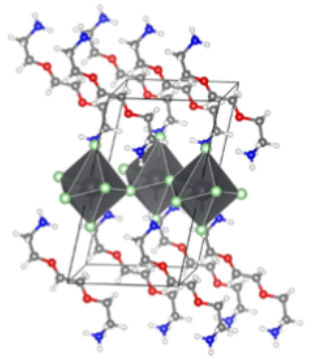

(EDBE) $\mathrm{PbCl}_{4}$ (c)

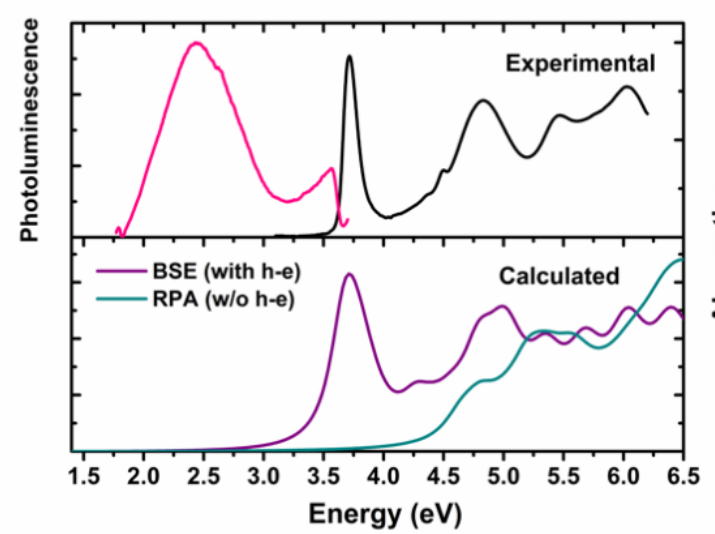

(d)

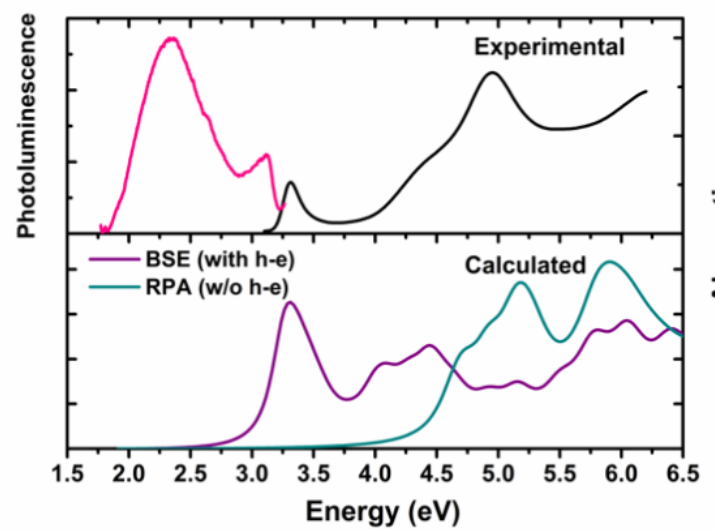

(e)
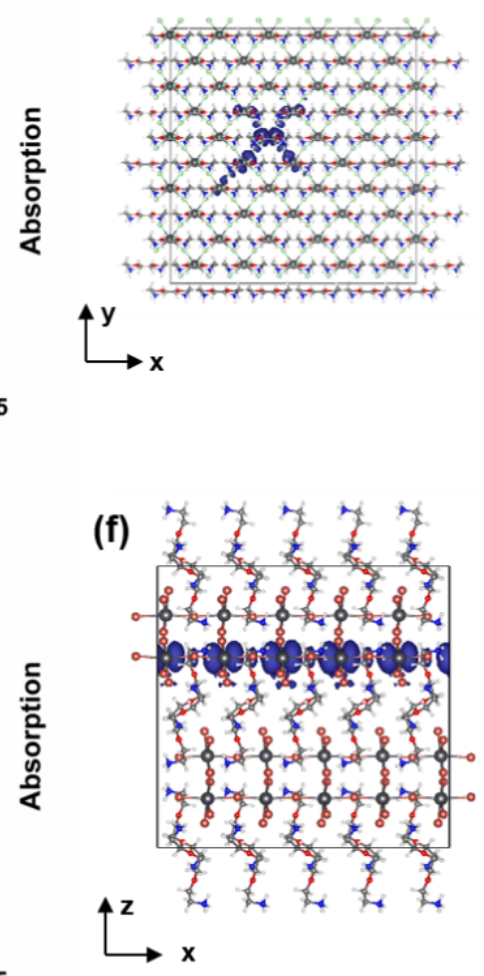

Exciton Wavefunction

(EDBE) $\mathrm{PbBr}_{4}$

Figure 1. Crystallographic, optical, and excitonic characteristics of the two-dimensional (EDBE) $\mathrm{PbCl}_{4}$ and (EDBE) $\mathrm{PbCl}_{4}$ hybrid perovskites: $(\mathrm{a}, \mathrm{b})$ crystal structures; $(\mathrm{c}, \mathrm{d})$ experimental absorption and photoluminescence spectra at room temperature (upper panel), and calculated absorption spectra with and without consideration of hole-electron (h-e) interactions (bottom panel); and (e, f) illustration of the exciton wavefunctions corresponding to the excitonic peaks.

After optimizing the crystal structures of the 2D hybrid perovskites based on their experimental lattice parameters [(EDBE)PbCl4: $a=7.73 \AA, b=7.54 \AA$ and $c=13.29 \AA$; (EDBE)PbBr $4: a=6.09 \AA$, $b=8.89 \AA$ and $c=28.78 \AA$ ], we calculated their band structures (see Figures S1-S2 in the Supporting Information) and compared their bandgaps using different methodologies (see Figure S3). The general gradient approximation (GGA)/Perdew-Burke-Ernzerhof (PBE) functional 
without consideration of spin-orbit coupling (SOC), $\mathrm{PBE} /$ woSOC, has been reported to reproduce well the bandgaps of 3D perovskites (e.g., $\mathrm{MAPbI}_{3}$ and MASnI 3 ), as a result of error cancellations,27 while PBE with SOC retains the band curvatures but changes the conduction band degeneracies and largely reduces the bandgaps. In our case, even when using PBE/woSOC, the bandgaps of the 2D hybrid perovskites remain underestimated. Therefore, we have used both the Heyd-Scuseria-Ernzerhof (HSE)28-29 hybrid functional implemented in VASP $30-31$ and the PBE functional together with the $\mathrm{G}_{0} \mathrm{~W}_{0}$ method implemented in Quantum Espresso (QE) $)_{32} / \mathrm{YAMBO}_{33}$ to correct the bandgaps based on the calculated results with account of SOC effects. We find that (EDBE)PbCl 4 has a direct bandgap [HSE+SOC: $3.27 \mathrm{eV} ; \mathrm{PBE}+\mathrm{G}_{0} \mathrm{~W}_{0}: 3.88$ $\mathrm{eV}$ ] at the $\Gamma$ point, while (EDBE) $\mathrm{PbBr} 4$ has a direct bandgap [HSE+SOC: $2.74 \mathrm{eV} ; \mathrm{PBE}+\mathrm{G}_{0} \mathrm{~W}_{0}$ : $3.45 \mathrm{eV}]$ at the A point of their respective Brillouin zones. The projected densities of states underline that the dominant contributions to the conduction and valence bands around the Fermi level are provided by the inorganic framework (i.e., $\mathrm{Pb}-6 s, 6 \mathrm{p}, \mathrm{Cl}-3 p$, or $\mathrm{Br}-4 p$ ); the organic EDBE ligands have no direct contributions to the electronic states in this region, which is similar to the case of the 3D Pb-based hybrid perovskites.

Figures 1c-d also compare the calculated optical absorption spectra with the experimental data obtained from the 2D hybrid perovskite films. Starting from the electronic wavefunctions of the ground state derived at the $\mathrm{PBE} / \mathrm{SOC}$ level, the optically allowed interband transitions were calculated by using the random phase approximation (RPA) method and by solving the BetheSalpeter equation (BSE) (see details in the Computational Methods). With respect to the RPA method, which does not include explicit electron-hole interactions, the BSE method reproduces well the excitonic features based on both quasi-electron and quasi-hole states; the resulting 
absorption spectra agree well with the experimental data. Note that scissor-operator corrections $\left(0.15 \mathrm{eV}\right.$ for $(\mathrm{EDBE}) \mathrm{PbCl}_{4}$ and $0.12 \mathrm{eV}$ for $\left.(\mathrm{EDBE}) \mathrm{PbBr} 4\right)$ were applied to correct for the slightly underestimated energy gaps, which affords a better comparison with experiment. The calculated spectra indicate that the high-energy absorption continuum is due to inter-band electronic transitions around the $\Gamma$ point, with the main contributions coming from $\mathrm{Pb}_{2}+(6 s) \mathrm{Cl}-$ $(3 p) \rightarrow \mathrm{Pb}_{2}+(6 p)$ and $\mathrm{Pb}_{2}+(6 s) \mathrm{Br}-(4 p) \rightarrow \mathrm{Pb}_{2}+(6 p)$. Although the organic EDBE cations do not directly contribute to these inter-band electronic transitions, they indirectly influence the electronic structure of the layered perovskites through crystal-structure effects, such as tilting and deformations of the inorganic framework. Moreover, quantum confinement within the 2D perovskite layers increases the strength of the electron-hole interactions, and localizes the excitons within the 2D perovskite planes (see Figures 1e-f); the large exciton binding energies measured experimentally are thus related to the strong localization calculated for the exciton wavefunctions.

When an electron is added to ionic and highly polar crystals such as hybrid perovskites, 20,34 the strong Coulomb interactions between the excess charge and the lattice ions enhances the electron-vibration couplings. In this case, electrons can self-trap over several lattice sites, forming so-called large polarons (also known as Fröhlich polarons).35-36 For example, in 3D hybrid perovskites such as $\mathrm{CH}_{3} \mathrm{NH}_{3} \mathrm{PbI}_{3}$, the motions of the organic cations, particularly their reorientations and the associated dipole fluctuations, can stabilize and localize charge carriers with the polarization cloud spanning many unit cells, thus forming large polarons.20, 37 Conversely, when excess electrons are spatially confined to a volume of approximately one unit cell or less, they correspond to so-called small polarons. In this situation, the short-range 
deformation potential due to the changes in local bonding related to the excess charge, plays a dominant role. Recently, Neukirch et al. demonstrated that localized charged states strongly couple to local structural lattice distortions in $\mathrm{MAPbI}_{3}$, with the $\mathrm{CH}_{3} \mathrm{NH}_{3}+$ configurations helping in creating small polaron states;19 the slow photocurrent degradation and fast self-healing in perovskite solar-cell devices were further attributed to the resulting charge accumulation.38 Thus, in 3D hybrid perovskites, both polar long-range interactions and short-range elastic local-field fluctuations play a role in the generation of large or small polarons. Similarly, the formation of polaronic states through deformation potentials and short-range interactions is expected to occur in 2D hybrid perovskites as well, through local volumetric strain and local molecular arrangements.

To simulate the photogeneration of small polarons and charge self-trapping effects in 2D hybrid perovskites, we started by constructing periodic models where we exerted local perturbations onto the $2 \mathrm{D}$ perovskite supercells. Selected bond lengths ( $\mathrm{Pb}-\mathrm{Pb}, \mathrm{Pb}-\mathrm{X}$ and $\mathrm{X}-\mathrm{X})$ were shortened in an ad-hoc manner to create the local structural deformations (i.e., the self-trapped charged states). Figure 2 shows the resulting energy levels and electronic charge densities at the valence band maximum (VBM) and conduction band minimum (CBM) for the unperturbed and perturbed systems. In the unperturbed (EDBE) $\mathrm{PbCl}_{4}$ and $(\mathrm{EDBE}) \mathrm{PbBr} 4$ systems, the charge densities at VBM and CBM are highly delocalized along the whole inorganic plane. Once a Pb$\mathrm{Pb}$ length is shortened, the CBM energy stabilizes and the electron density localizes at the $\mathrm{Pb}-\mathrm{Pb}$ sites, while the hole density at VBM is nearly unaffected. This $\mathrm{Pb}-\mathrm{Pb}$ dimerization causes the formation of a self-trapped electron at $\mathrm{Pb} 23+$ sites, in good agreement with previous experimental observations in lead halides.39-41 In the case of shortened X-X bond lengths, the hole density is 
highly localized at the $\mathrm{Cl}-\mathrm{Cl}$ or $\mathrm{Br}-\mathrm{Br}$ pairs, indicating the formation of $\mathrm{V}_{\mathrm{k}}$ centers, e.g., $\mathrm{Cl}_{2}$ - and $\mathrm{Br}_{2}$ - for (EDBE) $\mathrm{PbCl}_{4}$ and (EDBE) $\mathrm{PbBr}$, respectively. In the instance of shortened $\mathrm{Pb}-\mathrm{X}$ bond, holes localize at a single $\mathrm{Pb}$ atom, leading to the formation of $\mathrm{Pb}_{3+}$ centers coupled with the lattice deformation. Thus, the strong interaction between charge carriers and phonons yields the formation of self-trapped electron $\left(\mathrm{Pb}_{23+}\right)$ states and self-trapped hole $\left(\mathrm{Pb}_{3+}\right.$ and $\left.\mathrm{X}_{2-}\right)$ states.
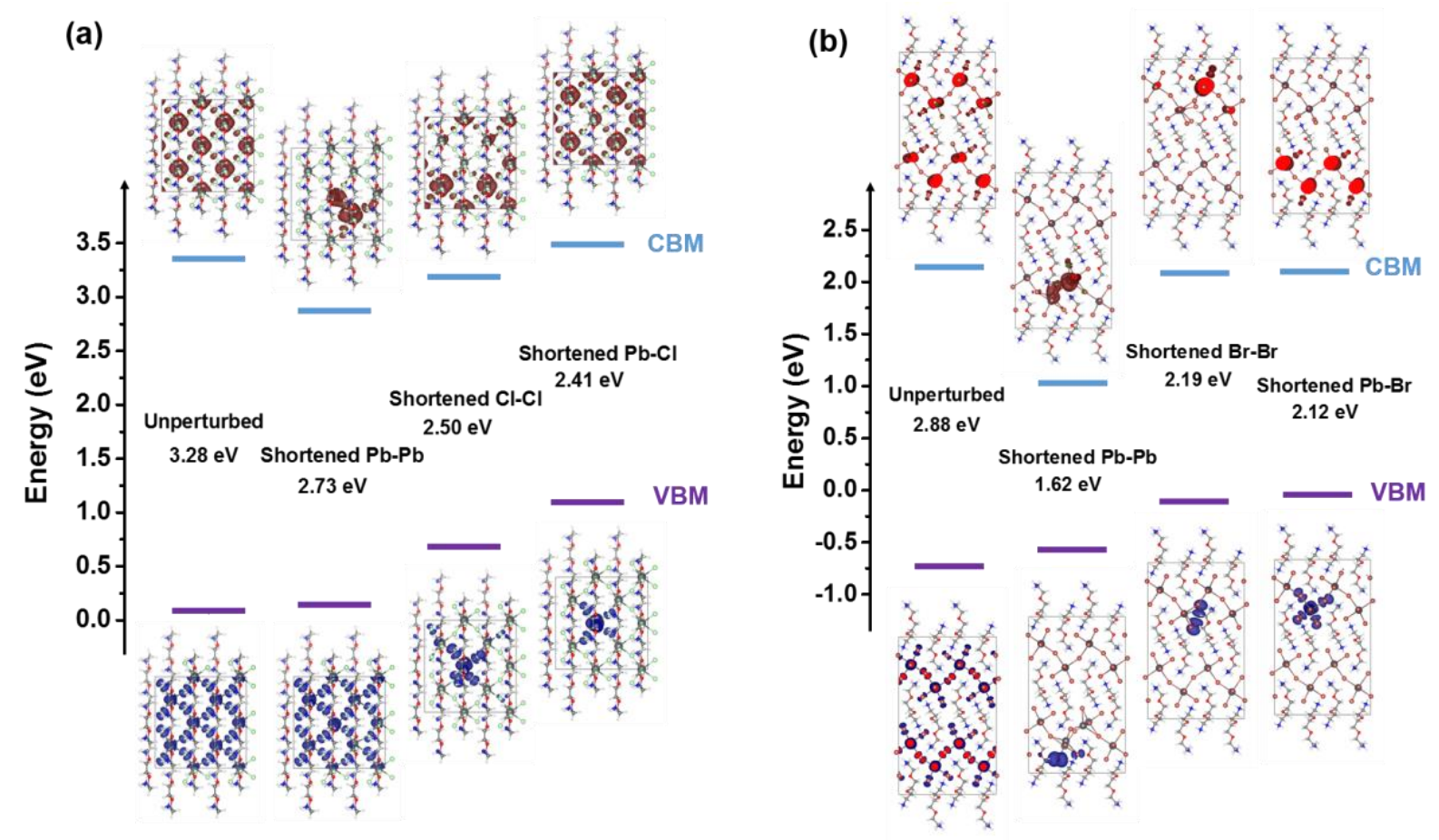

Figure 2. Energy levels marking the valence band maximum (VBM) and conduction band minimum (CBM) calculated at the HSE/SOC level, and corresponding charge density distributions for $2 \times 2 \times 1$ (a) (EDBE) $\mathrm{PbCl}_{4}$ and (b) (EDBE) $\mathrm{PbBr}_{4}$ supercells without and with perturbations: $i$ ) shortened $\mathrm{Pb}-\mathrm{Pb}$ distances; ii) shortened $\mathrm{Pb}-\mathrm{X}$ distances; and iii) shortened $\mathrm{X}-\mathrm{X}$ distances. 
These localized polaronic states are expected to act as emissive states within the gaps; the corresponding polaronic exciton energies are 2.73, 2.49, and 2.41 eV for (EDBE)PbCl4, and 1.62, 2.19, and $2.12 \mathrm{eV}$ for (EDBE) $\mathrm{PbBr} 4$, as evaluated at the HSE/SOC level. The spacing between these levels (0.3-0.4 eV) agrees well with the energetics of the three emissive states to which we previously attributed the white-light emission.17 Thus, upon resonant photoexcitation of the excitonic band, luminescence from these states gives rise to broadband, Stokes-shifted emission. Although the organic layers are not directly involved in polaron formation, charge and dielectric confinement in the layered structure significantly increases the carrier effective masses, strengthening electron-phonon coupling and facilitating polaron formation even at room temperature. Thus, the strong electron-phonon coupling is responsible for the formation of small polarons, which results in the exceptionally large Stokes shifts observed experimentally and further broadens the overall width of the radiative transitions.

To fully characterize the actual small polaron distributions in the $2 \mathrm{D}$ perovskites once a charge is removed from or added into the inorganic framework, we turned to the consideration of a molecular-cluster model for the 2D hybrid perovskites and applied the following methodology. We started with the optimized crystal structure of bulk (EDBE) $\mathrm{PbCl}_{4}$ and took a single inorganic layer surrounded by two layers of organic EDBE cations (see Figure S4). Then, we replaced the outer $\mathrm{NH}_{3}+$ terminations with $\mathrm{NH}_{2}$ in order to obtain a neutral single-layer slab that has electronic features similar to those in the bulk. The molecular clusters used for the charged-state calculations were then generated from this single layer slab following the rule $6 n \mathrm{~Pb}=n \times 1+2 n \times 2$ and $2 n \mathrm{~Pb}+n \mathrm{EDBE}=n \times 1+n \times 2$ in order to balance the charge of the molecular clusters $(n \mathrm{~Pb}, n \mathrm{EDBE}$, and $n \mathrm{x}$ are the number of $\mathrm{Pb}, \mathrm{EDBE}$, and $\mathrm{Cl}$ or $\mathrm{Br}$ atoms; $\mathrm{X} 1$ is a halogen atom that is only 
bonded to a single $\mathrm{Pb}$ atom, while $\mathrm{X} 2$ corresponds to a halogen atom that is bonded to two $\mathrm{Pb}$ atoms). Finally, we further simplified the cluster model by replacing the long chain EDBE cations with $\mathrm{CH}_{3} \mathrm{NH}_{3}+$ to make the evaluation of these clusters (containing hundreds of atoms) less computationally challenging, while retaining the electronic properties of the inorganic framework. The resulting (EDBE) $\mathrm{PbCl}_{4}$ cluster corresponds to $\left(\mathrm{CH}_{3} \mathrm{NH}_{3}\right)_{32} \mathrm{~Pb}_{12} \mathrm{Cl}{ }_{56}$ (see Figure 3a). The same computational strategy was adopted to generate the Br-based cluster, $\left(\mathrm{CH}_{3} \mathrm{NH}_{3}\right)_{17} \mathrm{~Pb}_{6} \mathrm{Br}_{29}$, from the (EDBE) $\mathrm{PbBr}_{4}$ crystal.

(a) Molecular Orbitals

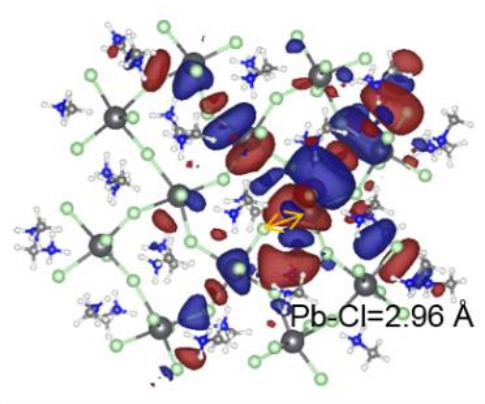

LUMO

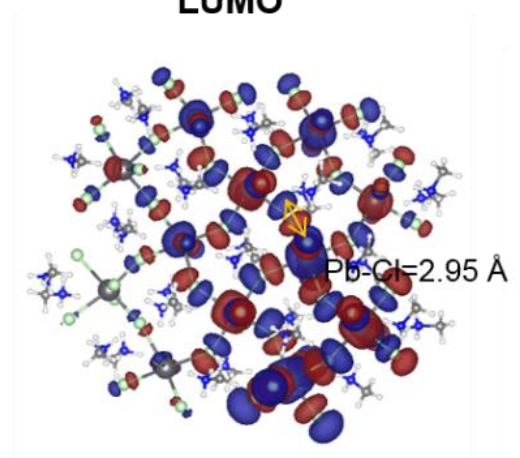

HOMO
Spin Density

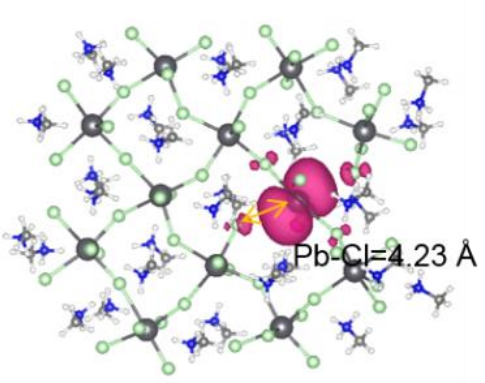

Electron

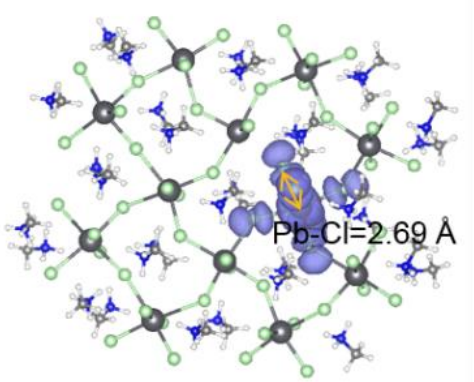

Hole (b)
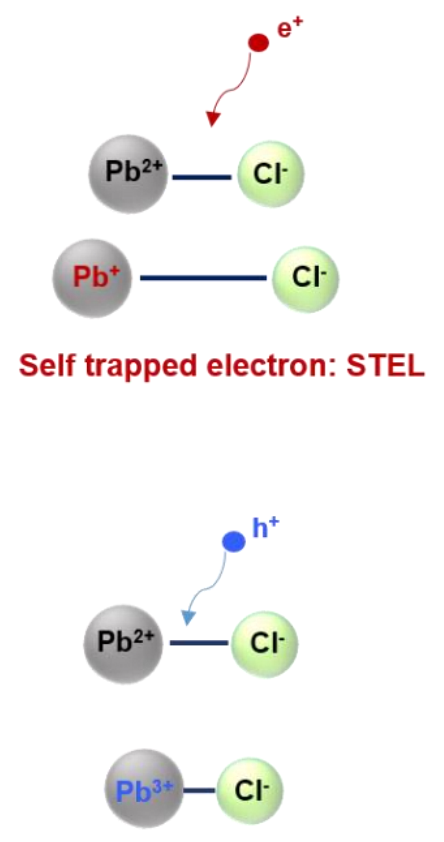

Self trapped hole: STH

Figure 3. (a) Charge densities in the frontier molecular orbitals and spin densities of the $(\mathrm{EDBE})_{32} \mathrm{~Pb}_{12} \mathrm{Cl}_{56}$ cluster. (b) Schematics of formation of self-trapped electron and hole. 
The charge densities in the frontier molecular orbitals (the highest occupied molecular orbital, HOMO, and the lowest unoccupied molecular orbital, LUMO) as well as the polaron charge (spin) densities obtained from the optimized geometries of the $\left(\mathrm{CH}_{3} \mathrm{NH}_{3}\right)_{32} \mathrm{~Pb}_{12} \mathrm{Cl}_{56}$ molecular cluster, are shown in Figure 3a. In the neutral system, both electrons and holes are largely delocalized within the inorganic plane. Once one electron is removed from the cluster, a $\mathrm{Pb}-\mathrm{Cl}$ bond shortens from 2.95 to $2.69 \AA$, and the hole density localizes at this $\mathrm{Pb}$ site and neighboring $\mathrm{Cl}$ atoms. The resulting charge density distribution is similar to the one obtained via the local perturbation of the supercell model when shortening the $\mathrm{Pb}-\mathrm{Cl}$ bond length (Figure 2), which confirms that the local structural change induces the formation of a localized, positive small polaron $\left(\mathrm{Pb}_{3}+\right.$ self-trapped hole). Adding an electron to the neutral cluster causes a very strong elongation of a Pb-Cl bond (from 2.96 to $4.23 \AA$ ), as the strongly localized additional electron density surrounding $\mathrm{Pb}_{2}+$ leads to large repulsive interactions between adjacent $\mathrm{Pb}$ and $\mathrm{Cl}$ atoms, thus markedly deforming the local geometry of the cluster.

To minimize possible edge effects in the cluster calculations, we also derived the molecular orbitals and spin densities in larger $\left(\mathrm{CH}_{3} \mathrm{NH}_{3}\right)_{52} \mathrm{~Pb}_{21} \mathrm{Cl}_{94}$ and $\left(\mathrm{CH}_{3} \mathrm{NH}_{3}\right)_{31} \mathrm{~Pb}_{12} \mathrm{Br} 55$ clusters, which are shown in Figure 4. While the charge densities are more delocalized throughout the inorganic framework in the large neutral clusters, similar polaron charge (spin) density features are calculated for both excess hole and electron, mainly spanning over a $\mathrm{Pb}$ atom and the nearest neighbor $\mathrm{Cl}$ atoms with a small radius of $r \sim 6 \AA$. In addition, in both cases, the electron densities are localized on two neighboring $\mathrm{Pb}$ atoms, indicating that negative charged species associated to $\mathrm{Pb}_{23}+$ are generated once an electron is added to the neutral clusters. 
The precise characterization of the actual cluster distortions induced by the presence of small polarons allow us to obtain a quantitative estimate of the polaron binding energy ( $\left.E_{\text {polaron }}\right)$, which is defined as the energy difference between the charged states at the neutral geometry and at the relaxed charged geometry. The resulting positive [negative] polaron binding energies are $E_{p o l a r o n}$ $=884[1240] \mathrm{meV}$ for the $\left(\mathrm{CH}_{3} \mathrm{NH}_{3}\right)_{52} \mathrm{~Pb}_{21} \mathrm{Cl}_{94}$ cluster and $E_{\text {polaron }}=392[1041] \mathrm{meV}$ for the $\left(\mathrm{CH}_{3} \mathrm{NH}_{3}\right)_{31} \mathrm{~Pb}_{12} \mathrm{Br}_{55}$ clusters (note that we did not include SOC effects in the cluster calculations, which may slightly underestimate the polaron binding energies42); the significantly larger negative polaron binding energies are related to the marked increase in the $\mathrm{Pb}-\mathrm{X}$ bond lengths in the negatively charged clusters. Such large polaron binding energies are consistent with the large Stokes shift observed in white-emitting 2D perovskites; they further confirm that the inorganic framework undergoes a strong structural relaxation upon generation of positive/negative polarons. 


\section{(a) $\quad(\mathrm{EDBE})_{52} \mathrm{~Pb}_{21} \mathrm{Cl}_{94}$}

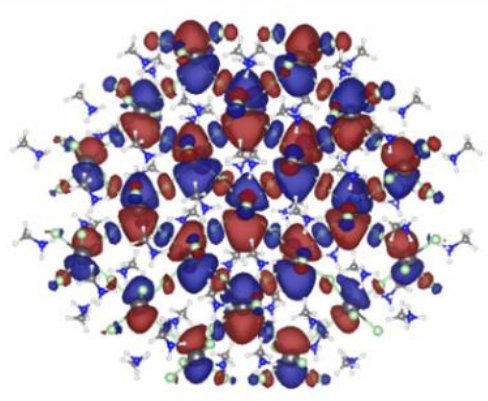

LUMO

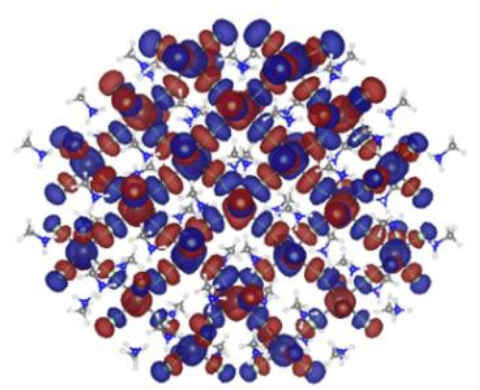

HOMO

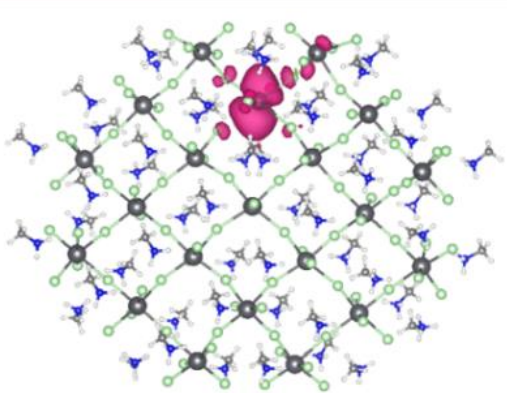

Electron

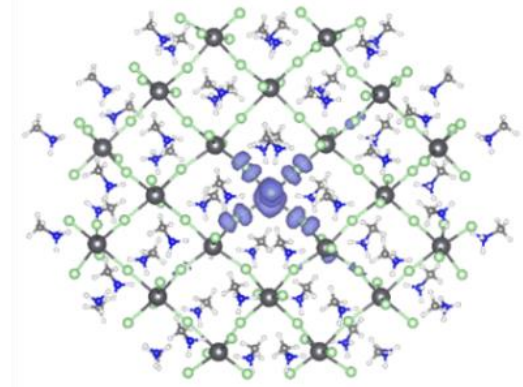

Hole

(b)

\section{$(\mathrm{EDBE})_{31} \mathrm{~Pb}_{12} \mathrm{Br}_{55}$}

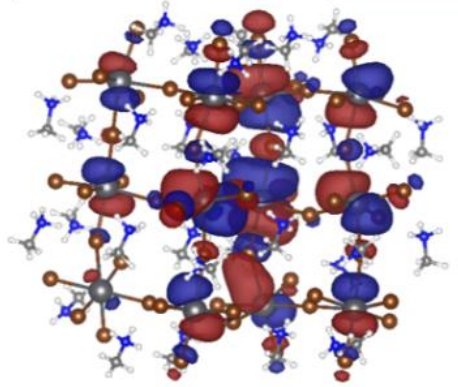

LUMO

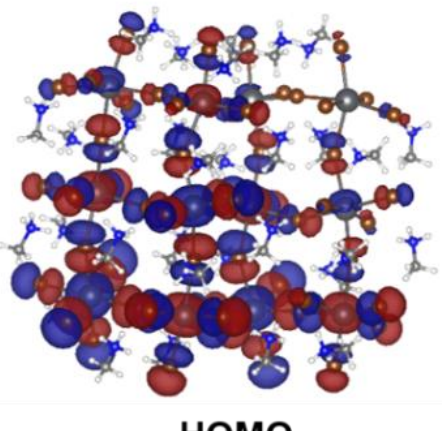

HOMO

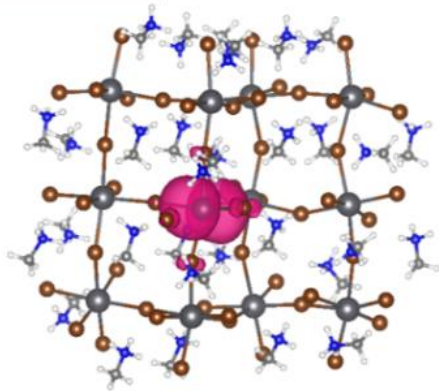

Electron

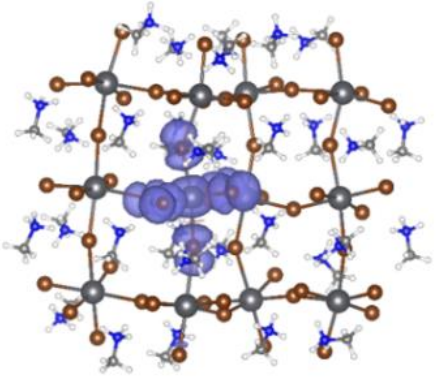

Hole

Figure 4. Charge densities in the frontier molecular orbitals and polaron charge (spin) densities for: (a) the $\left(\mathrm{CH}_{3} \mathrm{NH}_{3}\right)_{52} \mathrm{~Pb}_{21} \mathrm{Cl}_{94}$ cluster; and (b) $\left(\mathrm{CH}_{3} \mathrm{NH}_{3}\right)_{31} \mathrm{~Pb}_{12} \mathrm{Br} 55$ cluster. 
In summary, we have systematically studied the excitonic and polaronic properties of $2 \mathrm{D}$ hybrid perovskites with broadband emission via first-principles calculations. The optical absorption spectra of the 2D hybrid perovskites, which consist of excitonic features and interband transitions, are well reproduced when considering explicitly both electron-hole interactions and spin-orbit couplings. The formation of small polarons can be simulated by exerting local deformations of the perovskite supercells, which leads to self-trapped states with energy spacings on the order of $0.3-0.4 \mathrm{eV}$; these calculated spacings are consistent with the broadband emission spectra observed experimentally.

To reach a quantitative description of the polaron charge distributions and relaxation energies, we developed a (computationally intensive) molecular-cluster approach. The results point to the formation of small positive/negative polarons with large binding energies (Epolaron 0.4-1.2 eV), and tightly localized $(r \sim 6 \AA)$ at specific $\mathrm{Pb}-\mathrm{X}$ sites. These theoretical methodologies are applicable to other low-dimensional hybrid perovskites with strong electron-vibration interactions in order to investigate their charge localization and transport behavior. Our results suggest that broadband white-light emission can be achieved in various classes of lowdimensional hybrid perovskites with strong electron-vibration couplings and large photo-induced distortions of the crystalline lattice when using long organic cations. 
Computational Methods

Periodic Structure Calculations: Density functional theory (DFT) calculations were performed to optimize the crystal structures of (EDBE) $\mathrm{PbCl}_{4}$ and (EDBE) $\mathrm{PbBr}_{4}$ using the projectoraugmented wave (PAW) method as implemented in the VASP code.30-31 The experimental crystal structures of both crystals at room temperature were used as starting point. To obtain the electronic band structures and charge densities of the unperturbed and perturbed supercells in Figure 2, the Perdew-Burke-Ernzerhof (PBE) functional, with and without consideration of spinorbit coupling (SOC), as well as the hybrid functional Heyd-Scuseria-Ernzerhof (HSE)28-29 were used. The plane-wave cutoff energy was set to $500.0 \mathrm{eV}$ and $\Gamma$-centered $6 \times 6 \times 6$ and $6 \times 6 \times 2$ $K$-meshes in the Brillouin zone were employed for (EDBE) $\mathrm{PbCl}_{4}$ and (EDBE)PbBr4, respectively. The bulk crystal structures were fully relaxed until the total force on each atom was $<0.01 \mathrm{eV} / \AA_{-1}$. The molecular graphics viewer (VESTA) was used to plot all the crystal/molecular structures and charge densities.

Cluster Structure Calculations: The neutral and charged geometries of the 2D hybrid perovskite clusters were optimized at the hybrid range-corrected functional CAM-B3LYP level with the Gaussian 09 code, version D.01; the LANL2DZ basis set was used for the $\mathrm{Pb}$ atoms and the 6$31 \mathrm{G}^{*}$ basis set was used to describe $\mathrm{H}, \mathrm{C}, \mathrm{N}, \mathrm{O}, \mathrm{Cl}$ and $\mathrm{Br}$. To mimic the highly polarizable dielectric environment of the perovskites, we considered a dielectric medium $(\varepsilon=78.35)$ via the conductor-like polarizable continuum model (CPCM).

Optical Response Calculations: The ground-state Kohn-Sham energies and wavefunctions were obtained with the PBE functional using the PWSCF code implemented in the Quantum 
ESPRESSO package.32 Norm-conserving Troullier-Martins pseudopotentials including fullrelativistic effects were used to describe the electron-ion interactions. Plane-wave basis set cutoffs of the wave functions and the augmented density were set at 80 and $480 \mathrm{Ry}$, respectively. The $4 \times 4 \times 4$ and $4 \times 2 \times 4$ Monkhorst-Pack grids were chosen for sampling the Brillouin zone of (EDBE) $\mathrm{PbCl}_{4}$ and (EDBE) $\mathrm{PbBr}_{4}$, respectively. Based on the electronic wavefunctions of the ground state, the optical transitions were calculated using the Bethe-Salpeter equations (BSE) method implemented in the YAMBO code: 33

$$
\left(E_{c \mathbf{k}}-E_{v \mathbf{k}}\right) A_{v c \mathbf{k}}^{S}+\sum_{\mathbf{k} / v \prime c \prime}\left\langle v c \mathbf{k}\left|K_{e h}\right| v^{\prime} c^{\prime} \mathbf{k}^{\prime}\right\rangle A_{v \prime c / \mathbf{k}^{\prime}}^{S}=\Omega^{S} A_{v c \mathbf{k}}^{S}
$$

where $E_{c \mathbf{k}}$ and $E_{v \mathbf{k}}$ are the quasiparticle energies of the conduction and valence states, respectively; $A_{v c \mathbf{k}}^{S}$ are the expansion coefficients of the excitons and $\Omega^{S}$ are the eigenenergies.

The imaginary part $(\varepsilon 1)$ of the optical response was calculated by evaluating direct electronic transitions between occupied and higher-energy unoccupied electronic states as obtained from

$$
\varepsilon_{1}(\omega) \propto \sum_{S}\left|\sum_{c v \mathbf{k}} A_{v c \mathbf{k}}^{S} \frac{\left\langle c \mathbf{k}\left|p_{i}\right| v \mathbf{k}\right\rangle}{\epsilon_{c \mathbf{k}}-\epsilon_{v \mathbf{k}}}\right| \delta\left(\Omega^{S}-\hbar \omega-\Gamma\right)
$$

where $\left\langle c \mathbf{k}\left|p_{i}\right| v \mathbf{k}\right\rangle$ are the dipole matrix elements for electronic transitions from valence to conduction states.

The absorption coefficient was obtained as:

$$
\alpha=2 \omega\left[\frac{\left(\varepsilon_{1}^{2}(\omega)+\varepsilon_{2}^{2}(\omega)\right)^{1 / 2}-\varepsilon_{1}(\omega)}{2}\right]^{1 / 2}
$$


SOC interactions and the spinor wave functions were also included as input for the optical response calculations. This approach has been previously used to reproduce successfully the optical properties of hybrid perovskite systems with spin-orbit interactions. 43

\section{ASSOCIATED CONTENT}

Supporting Information. Illustrations of the band structures and band gaps calculated by different methods and codes and of how the 2D perovskite clusters were built. The Supporting Information is available free of charge on the ACS Publications website.

\section{AUTHOR INFORMATION}

\section{Corresponding Author}

*E-mail: jean-luc.bredas@kaust.edu.sa.

\section{ORCID}

Jean-Luc Brédas: 0000-0001-7278-4471

\section{Notes}

The authors declare no competing financial interest.

\section{ACKNOWLEDGMENTS}

This work was supported by King Abdullah University of Science and Technology (KAUST) and by the Singapore National Research Foundation (NRF-CRP14-2014-03). We gratefully thank the IT Research Computing Team and Supercomputing Laboratory at KAUST for computational and storage resources as well as precious assistance. 


\section{REFERENCES}

(1) Kojima, A.; Teshima, K.; Shirai, Y.; Miyasaka, T. Organometal Halide Perovskites as Visible-Light Sensitizers for Photovoltaic Cells. J. Am. Chem. Soc. 2009, 131, 6050-6051.

(2) Snaith, H. J. Perovskites: The Emergence of a New Era for Low-Cost, High-Efficiency Solar Cells. J. Phys. Chem. Lett. 2013, 4, 3623-3630.

(3) Li, X.; Bi, D. Q.; Yi, C. Y.; Decoppet, J. D.; Luo, J. S.; Zakeeruddin, S. M.; Hagfeldt, A.; Gratzel, M. A Vacuum Flash-Assisted Solution Process for High-Efficiency Large-Area Perovskite Solar Cells. Science 2016, 353, 58-62.

(4) Tsai, H. H.; Nie, W. Y.; Blancon, J. C.; Toumpos, C. C. S.; Asadpour, R.; Harutyunyan, B.; Neukirch, A. J.; Verduzco, R.; Crochet, J. J.; Tretiak, S. et al. High-Efficiency TwoDimensional Ruddlesden-Popper Perovskite Solar Cells. Nature 2016, 536, 312-316.

(5) Mitzi, D. B. Synthesis, Structure, and Properties of Organic - Inorganic Perovskites and Related Materials, Progress in Inorganic Chemistry; John Wiley \& Sons, Inc., 2007, 1-121.

(6) Saparov, B.; Mitzi, D. B. Organic-Inorganic Perovskites: Structural Versatility for Functional Materials Design. Chem. Rev. 2016, 116, 4558-4596.

(7) Mitzi, D. B. Hybrid Organic-Inorganic Electronics. In Functional Hybrid Materials; WileyVCH Verlag GmbH \& Co. KGaA: Weinheim, Germany, 2005, 347-386.

(8) Ogawa, T.; Kanemitsu, Y. Optical Properties of Low-Dimensional Materials; World Scientific, 1996.

(9) Muljarov, E. A.; Tikhodeev, S. G.; Gippius, N. A.; Ishihara, T. Excitons in Self-Organized Semiconductor/Insulator Superlattices: PbI-Based Perovskite Compounds. Phys. Rev. B 1995, 51, 14370-14378.

(10) Li, Y. Y.; Lin, C. K.; Zheng, G. L.; Cheng, Z. Y.; You, H.; Wang, W. D.; Lin, J. Novel $<110>$-Oriented Organic-Inorganic Perovskite Compound Stabilized by N-(3-Aminopropyl) Imidazole with Improved Optical Properties. Chem. Mater. 2006, 18, 3463-3469.

(11) Dohner, E. R.; Jaffe, A.; Bradshaw, L. R.; Karunadasa, H. I. Intrinsic White-Light Emission from Layered Hybrid Perovskites. J. Am. Chem. Soc. 2014, 136, 13154-13157.

(12) Yangui, A.; Garrot, D.; Lauret, J. S.; Lusson, A.; Bouchez, G.; Deleporte, E.; Pillet, S.; Bendeif, E. E.; Castro, M.; Triki, S. et al. Optical Investigation of Broadband White-Light Emission in Self-Assembled Organic-Inorganic Perovskite $\left(\mathrm{C}_{6} \mathrm{H}_{11} \mathrm{NH}_{3}\right)_{2} \mathrm{PbBr}_{4}$. J. Phys. Chem. C 2015, 119, 23638-23647. 
(13) Dohner, E. R.; Hoke, E. T.; Karunadasa, H. I. Self-Assembly of Broadband White-Light Emitters. J. Am. Chem. Soc. 2014, 136, 1718-1721.

(14) Ki, W.; Li, J. A Semiconductor Bulk Material That Emits Direct White Light. J. Am. Chem. Soc. 2008, 130, 8114-8115.

(15) Bowers, M. J.; McBride, J. R.; Rosenthal, S. J. White-Light Emission from Magic-Sized Cadmium Selenide Nanocrystals. J. Am. Chem. Soc. 2005, 127, 15378-15379.

(16) Hu, T.; Smith, M. D.; Dohner, E. R.; Sher, M. J.; Wu, X. X.; Trinh, M. T.; Fisher, A.; Corbett, J.; Zhu, X. Y.; Karunadasa, H. I. et al. Mechanism for Broadband White-Light Emission from Two-Dimensional (110) Hybrid Perovskites. J. Phys. Chem. Lett. 2016, 7, 2258-2263.

(17) Cortecchia, D.; Yin, J.; Bruno, A.; Lo, S.-Z. A.; Gurzadyan, G. G.; Mhaisalkar, S.; Brédas, J.-L.; Soci, C. Polaron Self-Localization in White-Light Emitting Hybrid Perovskites. 2016, arXiv:1603.01284.

(18) Wehrenfennig, C.; Liu, M. Z.; Snaith, H. J.; Johnston, M. B.; Herz, L. M. Homogeneous Emission Line Broadening in the Organo Lead Halide Perovskite $\mathrm{CH}_{3} \mathrm{NH}_{3} \mathrm{PbI}_{3}-\mathrm{xCl}$. $J$. Phys. Chem. Lett. 2014, 5, 1300-1306.

(19) Neukirch, A. J.; Nie, W. Y.; Blancon, J. C.; Appavoo, K.; Tsai, H.; Sfeir, M. Y.; Katan, C.; Pedesseau, L.; Even, J.; Crochet, J. J. et al. Polaron Stabilization by Cooperative Lattice Distortion and Cation Rotations in Hybrid Perovskite Materials. Nano Lett. 2016, 16, 38093816.

(20) Zhu, X. Y.; Podzorov, V. Charge Carriers in Hybrid Organic-Inorganic Lead Halide Perovskites Might Be Protected as Large Polarons. J. Phys. Chem. Lett. 2015, 6, 4758-4761.

(21) Gauthron, K.; Lauret, J. S.; Doyennette, L.; Lanty, G.; Al Choueiry, A.; Zhang, S. J.; Brehier, A.; Largeau, L.; Mauguin, O.; Bloch, J. et al. Optical Spectroscopy of TwoDimensional Layered $\left(\mathrm{C}_{6} \mathrm{H}_{5} \mathrm{C}_{2} \mathrm{H}_{4}-\mathrm{NH}_{3}\right)_{2}-\mathrm{PbI}_{4}$ Perovskite. Opt. Express 2010, 18, 59125919.

(22) Maddalena, F.; Boix, P. P.; Mathews, N.; Soci, C.; Mhaisalkar, S. In Organic-Inorganic Halide Perovskite Photovoltaics; Springer, 2016, 201-222.

(23) Soufiani, A. M.; Huang, F. Z.; Reece, P.; Sheng, R.; Ho-Baillie, A.; Green, M. A. Polaronic Exciton Binding Energy in Iodide and Bromide Organic-Inorganic Lead Halide Perovskites. Appl. Phys. Lett. 2015, 107. 
(24) Kitazawa, N.; Watanabe, Y. Optical Properties of Natural Quantum-Well Compounds ( $\left.\mathrm{C}_{6} \mathrm{H}_{5}-\mathrm{C}_{\mathrm{n}} \mathrm{H}_{2 \mathrm{n}}-\mathrm{NH}_{3}\right)_{2} \mathrm{PbBr} 4(\mathrm{~N}=1-4)$. J. Phys. Chem. Solids 2010, 71, 797-802.

(25) Papavassiliou, G. C.; Mousdis, G. A.; Koutselas, I. B. Some New Organic-Inorganic Hybrid Semiconductors Based on Metal Halide Units: Structural, Optical and Related Properties. Adv. Mater. Opt. Electron. 1999, 9, 265-271.

(26) Gonzalez-Carrero, S.; Espallargas, G. M.; Galian, R. E.; Perez-Prieto, J. Blue-Luminescent Organic Lead Bromide Perovskites: Highly Dispersible and Photostable Materials. J. Mater. Chem. A 2015, 3, 14039-14045.

(27) Umari, P.; Mosconi, E.; De Angelis, F. Relativistic Gw Calculations on $\mathrm{CH}_{3} \mathrm{NH}_{3} \mathrm{PbI}_{3}$ and $\mathrm{CH}_{3} \mathrm{NH}_{3} \mathrm{SnI}_{3}$ Perovskites for Solar Cell Applications. Sci. Rep. 2014, 4, 4467.

(28) Heyd, J.; Scuseria, G. E.; Ernzerhof, M. Hybrid Functionals Based on a Screened Coulomb Potential. J. Chem. Phys. 2003, 118, 8207-8215.

(29) Paier, J.; Hirschl, R.; Marsman, M.; Kresse, G. The Perdew-Burke-Ernzerhof ExchangeCorrelation Functional Applied to the G2-1 Test Set Using a Plane-Wave Basis Set. J. Chem. Phys. 2005, 122, 234102.

(30) Kresse, G.; Furthmuller, J. Efficiency of Ab-Initio Total Energy Calculations for Metals and Semiconductors Using a Plane-Wave Basis Set. Comput. Mater. Sci. 1996, 6, 15-50.

(31) Kresse, G.; Joubert, D. From Ultrasoft Pseudopotentials to the Projector Augmented-Wave Method. Phys. Rev. B 1999, 59, 1758-1775.

(32) Giannozzi, P.; Baroni, S.; Bonini, N.; Calandra, M.; Car, R.; Cavazzoni, C.; Ceresoli, D.; Chiarotti, G. L.; Cococcioni, M.; Dabo, I. et al. Quantum Espresso: A Modular and OpenSource Software Project for Quantum Simulations of Materials. J. Phys. Condens. Matter 2009, 21, 395502.

(33) Marini, A.; Hogan, C.; Gruning, M.; Varsano, D. Yambo: An Ab Initio Tool for Excited State Calculations. Comput. Phys. Commun. 2009, 180, 1392-1403.

(34) Emin, D. Polarons; Cambridge University Press, U.K.; 2013.

(35) Feynman, R. P. Slow Electrons in a Polar Crystal. Phys. Rev. 1955, 97, 660-665.

(36) Frohlich, H. Electrons in Lattice Fields. Adv. Phys. 1954, 3, 325-361.

(37) Ma, J.; Wang, L. W. Nanoscale Charge Localization Induced by Random Orientations of Organic Molecules in Hybrid Perovskite $\mathrm{CH}_{3} \mathrm{NH}_{3} \mathrm{PbI}_{3}$. Nano Lett. 2015, 15, 248-253. 
(38) Nie, W.; Blancon, J. C.; Neukirch, A. J.; Appavoo, K.; Tsai, H.; Chhowalla, M.; Alam, M. A.; Sfeir, M. Y.; Katan, C.; Even, J. et al. Light-Activated Photocurrent Degradation and Self-Healing in Perovskite Solar Cells. Nat Commun 2016, 7, 11574.

(39) Nistor, S. V.; Schoemaker, D. Consistent Analysis and Tl-2(+) and Pb-2(3+) Center Esr Parameters. Physica Status Solidi B-Basic Research 1995, 190, 339-346.

(40) Nistor, S. V.; Goovaerts, E.; Schoemaker, D. Direct Observation of Electron Self-Trapping in $\mathrm{PbCl}_{2}$ Crystals. Phys. Rev. B 1993, 48, 9575-9580.

(41) Iwanaga, M.; Azuma, J.; Shirai, M.; Tanaka, K.; Hayashi, T. Self-Trapped Electrons and Holes in PbBr2 Crystals. Phys. Rev. B 2002, 65, 214306.

(42) Li, Z.; Ma, Z. S.; Wright, A. R.; Zhang, C. Spin-Orbit Interaction Enhanced Polaron Effect in Two-Dimensional Semiconductors. Appl. Phys. Lett. 2007, 90, 112103.

(43) Minaev, B. F.; Muldakhmetov, Z. M. Effect of Spin-Orbital Interaction on the Intensity of Optical Doublet-Doublet and Triplet-Triplet Transitions in Molecules. Opt. Spektrosk. 1984, 56, 48-52. 